\title{
EVALUACIÓN DE SITIOS WEB MULTILINGÜES: METODOLOGÍA Y HERRAMIENTA HEURÍSTICA
}

Mar Andreu-Vall y Mari-Carmen Marcos
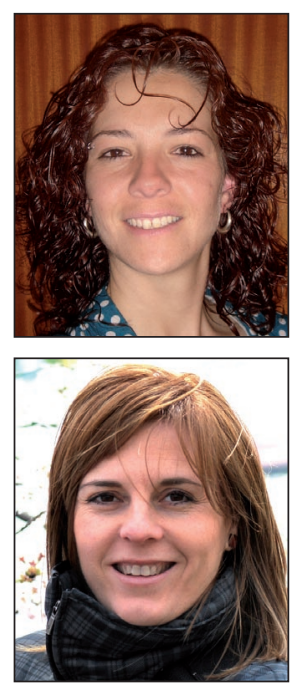

Mar Andreu-Vall es licenciada en traducción e interpretación por la Universidad Autónoma de Barcelona (2004) y titulada en el Máster en Gestión de Contenidos Digitales por la Universidad de Barcelona (2011). Desde 2004 ha trabajado como traductora autónoma. Actualmente es lingüista y coordinadora de proyectos de traducción web en AutomaticTrans, empresa que ofrece servicios y soluciones para proyectos lingüísticos multilingües.

AutomaticTrans SL Ronda Sant Pere, 13. 08010 Barcelona, España mmandreu@automatictrans.es

Mari-Carmen Marcos es profesora de la Universidad Pompeu Fabra de Barcelona, subdirectora del Máster Online en Documentación Digital y codirectora del Máster en Buscadores. También es profesora del Máster en Gestión de Contenidos UB-UPF. Es doctora y licenciada en documentación. Su línea de investigación se centra en la evaluación de interfaces como medio para la interacción entre las personas y la tecnología.

Universitat Pompeu Fabra, Departamento de Comunicación Roc Boronat, 138. 08018 Barcelona, España http://www.mcmarcos.com mcarmen.marcos@upf.edu

\section{Resumen}

Se propone una metodología de evaluación de sitios web dirigidos a un público multilingüe y multicultural. Para ello, se han revisado los estándares y recomendaciones de diversas fuentes validadas, se han creado unas pautas generales (heurísticas) y se han desarrollado indicadores detallados para ellas. El resultado es una checklist para la evaluación de sitios web con público internacional, tanto monolingües (con o sin internacionalización) como multilingües (con o sin localización).

\section{Palabras clave}

Usabilidad, Evaluación heurística, Localización, Internacionalización, Multilingüismo.

\section{Title: Multilingual websites evaluation: methodology and heuristic tool}

\section{Abstract}

A methodology for evaluating the suitability of websites to multilingual and multicultural audiences is proposed. Standards and validated recommendations have been checked in order to create a (heuristic) guideline. The result is a checklist for evaluating websites with international audiences, both monolingual (with or without globalization) and multilingual (with or without localization).

\section{Keywords}

Usability, Heuristic evaluation, Localization, Internationalization, Multilinguism.

Andreu-Vall, Mar; Marcos, Mari-Carmen. “Evaluación de sitios web multilingües: metodología y herramienta heurística”. El profesional de la información, 2012, mayo-junio, v. 21, n. 3, pp. 254-260.

\section{Introducción}

Las transformaciones relacionadas con el proceso de globalización de la sociedad y la economía han puesto de relieve la gran importancia estratégica que han adquirido las lenguas en la cultura, la cohesión social, la economía y el desarrollo de las sociedades. En este sentido, la presencia de las diferentes lenguas en la Web va adquiriendo progresivamente más peso, y a pesar de la predominancia del inglés y el chino, el multilingüismo permite que más usuarios puedan

Nota: Una versión preliminar de este estudio fue presentada en el congreso Interacción 2011. Puede consultarse en: Andreu, Mar; Marcos, MariCarmen. "Multilingüismo en la web. Pautas para un diseño centrado en el usuario". Interacción 2011: XII Congreso internacional de interacción persona-ordenador, Lisboa, 2-5 sept. 2011. ISBN: 9788492812349 
acceder a contenidos web en su lengua. En este panorama hay dos conceptos que merece la pena aclarar: internacionalización y localización.

La internacionalización (i18n) ${ }^{1}$ es un concepto que procede del área de la programación y que consiste en que el código esté preparado para poder desarrollar una aplicación para diversos idiomas. Aplicado al diseño web, se trata del desarrollo de un sitio o aplicación web que permita una fácil adaptación lingüística (localización) cuando va dirigido a un público con una lengua o cultura diferentes al idioma para el que se ha creado originalmente. Por otra parte, la localización (I10n) es el proceso de adaptación de un producto para adecuarlo a las necesidades, tanto lingüísticas como culturales, de un público destinatario concreto llamado público local. La localización engloba aspectos más complejos que la simple traducción del contenido, ya que incluye la adaptación de elementos como formatos numéricos, de fecha y de hora, etc.

En este artículo proponemos una metodología de evaluación de la adecuación de sitios web a un público multilingüe y multicultural. Para ello se han revisado los estándares y recomendaciones de diversas fuentes validadas, se han creado unas pautas generales (heurísticas) y se han desarrollado indicadores detallados para ellas. El resultado es un checklist para la evaluación de sitios web con público internacional, tanto monolingües (con o sin internacionalización) como multilingües (con o sin localización).

En la sección head del código debe incluirse una etiqueta meta de contenido en la que se indique que la codificación será de tipo UTF-8

\section{Metodología: la evaluación heurística}

Existen muchos criterios y puntos de vista desde los que analizar y evaluar un sitio web. En este estudio hemos utilizado una de las técnicas de evaluación centradas en el usuario denominada evaluación heurística. Consiste en que un grupo de expertos valore si los elementos de la interfaz del usuario de un sitio web o aplicación se corresponden con los principios de usabilidad establecidos a partir de una lista de principios o heurísticas. Esta técnica consigue detectar un gran número de problemas de usabilidad en sitios web con un menor coste que las técnicas que involucran usuarios (Nielsen, 1994).

Así como para la evaluación general de sitios web existen criterios muy establecidos y probados que proporcionan la seguridad de que se está evaluando todo lo que es necesario evaluar (como los de Nielsen y Molich, 1990, simplificados más tarde por Nielsen y Mack, 1994), estas pautas no cubren aspectos específicos, como el que ahora nos ocupa, que es el multilingüismo.

La evaluación que proponemos no pretende sustituir a la heurística tradicionalmente usada en sitios web, sino que es un complemento para aquellos sitios que sí son multilingües.
Para su elaboración se han tomado como punto de partida los principios heurísticos de Nielsen y la adaptación realizada posteriormente por Hassan y Martín (2003) y se han incorporado los estándares de localización y usabilidad de otros autores (Del Galdo, Nielsen, 1996; Nielsen, 2000; HiIlier, 2002), además de las pautas del World Wide Web Consortium (W3C).

El resultado es la guía de evaluación heurística para sitios web multilingües presentada en la sección 3.

No adecuar el formato de fecha puede provocar confusión a la hora de interpretar la información

\section{Pautas para la evaluación de la localización en sitios web multilingües}

Presentamos a continuación las 24 pautas definidas para la evaluación de un sitio web, estructuradas en 5 categorías. Para cada pauta se indica el impacto que tiene su falta de cumplimiento ( 1 = leve, es molesto pero no impide realizar la tarea que el usuario pretende; 2 = medio, dificulta la tarea; 3 = grave, impide realizar la tarea).

\section{Grupo 1. Aspectos relacionados con el código fuente}

Esta categoría evalúa los elementos del código html y su adecuación a las necesidades de la internacionalización. Se han tomado como fuente las siguientes pautas del W3C:

- Guía breve de internacionalización

- An introduction to multilingual web addresses

- Etiquetas de idioma en html y xml

- El tamaño del texto en la traducción

- Internationalization best practices: Specifying language in xhtml \& html content

Pautas:

\subsection{Codificación unicode en la web (charset UTF-8)}

En la sección head del código debe incluirse una etiqueta meta de contenido en la que se indique que la codificación será de tipo UTF-8 (8-bit Unicode transformation format). Así el navegador interpretará los caracteres de la mayoría de los alfabetos. Un ejemplo:

<meta http-equiv="Content-Type" content="text/html; charset=utf- 8 " $/>$

Impacto: grave (3)

1.2. Coherencia de la lengua de las urls de las diferentes versiones con la lengua en la que está redactado el contenido

Las palabras que se usen como parte de la url deben estar en el mismo idioma de la página a la que esa url dirige. Por lo tanto, un sitio web que tiene una página en 3 idiomas deberá adaptar también los nombres de las categorías, subcategorías y páginas para que estén traducidas a los 3 idiomas (figura 1).

Impacto: medio (2) 


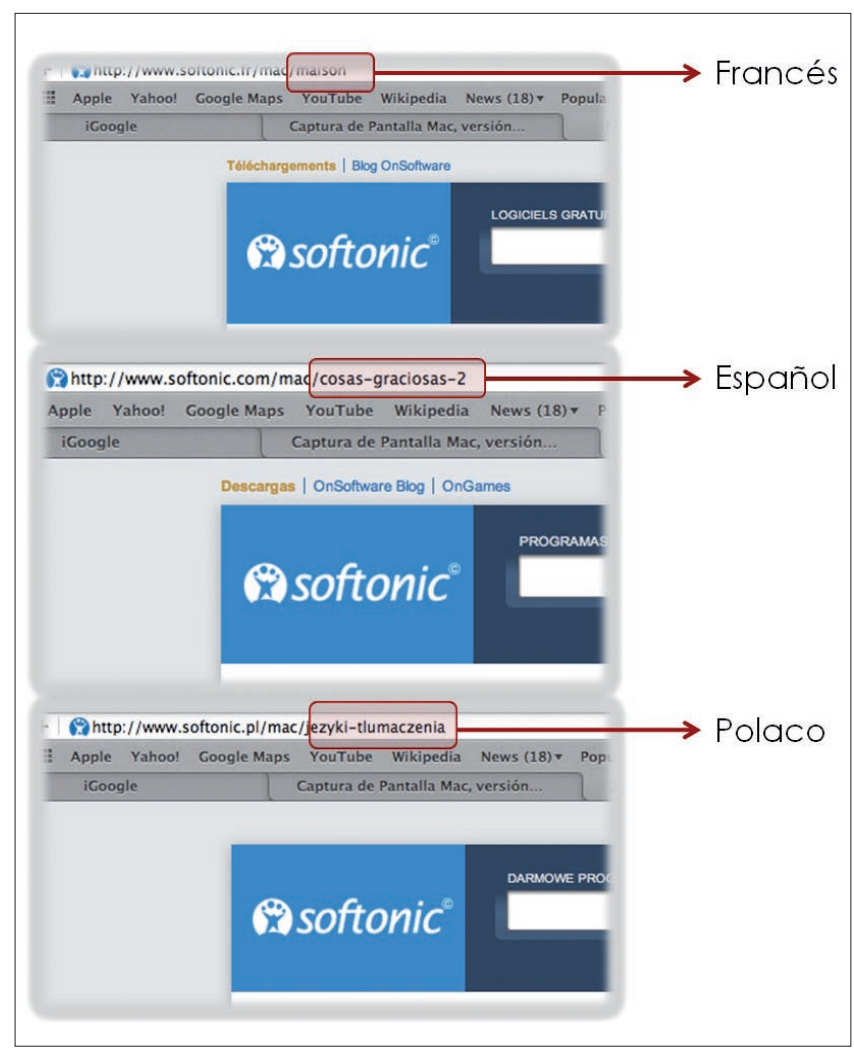

Figura 1. Ejemplo de urls traducidas

\subsection{Declaración del idioma del contenido en el encabeza- miento de cada página}

Al inicio del documento debe indicarse el idioma en el que está escrita la página. En caso de ser una variante del idioma para un país en concreto, se añade el país. El código sería así para español de España: <html lang="es">

Impacto: grave (3)

\subsection{Cambios de idioma del contenido dentro de una mis-} ma página

En caso de que alguna palabra, frase o párrafo dentro de una página web esté en un idioma distinto al del resto de la página, deberá indicarse en el código. Por ejemplo, si es una página en español y comienza un párrafo en francés, se indicará con la etiqueta de párrafo seguida del atributo de lenguaje: $\langle p$ lang=" $\mathrm{fr}$ ">

Impacto: grave (3)

\subsection{Expansión del texto}

Algunos idiomas se caracterizan por tener palabras muy largas. El maorí es conocido por ese rasgo, por ejemplo puede haber una palabra de 85 caracteres, pero sin ir más lejos, el alemán o el polaco son idiomas con palabras largas, lo que hace que necesiten más espacio en la página web. Por eso, al diseñar el sitio web hay que prever la posible expansión que tendrán las palabras al cambiar de lengua. Eso implica que el diseño debe permitir que los espacios sean flexibles, no cerrados, pues se puede encontrar que palabras muy cortas en inglés son mucho más largas en la mayoría de las lenguas (figura 2).

Impacto: leve (1)

\subsection{Cambio de lengua del nodo de los enlaces}

Es común incluir enlaces a páginas web que se encuentran en un idioma distinto al de la página en la que está el link. La buena práctica es indicar el cambio de idioma en el código en la misma etiqueta del enlace $(<a>)$ con el atributo href lang. Por ejemplo, en una página en español que contenga un enlace a una página en inglés, el código sería así:

$<$ a href=http://www.otrapagina.com/ hreflang="en">Otra página $</ a>$

Impacto: leve (1)

\section{El diseño visual usado en la versión di- rigida a un país puede que tenga que variar con respecto al que se use para público de otros países}

\section{Grupo 2. Aspectos relativos a la localización lingüís- tica}

Esta categoría tiene como objetivo valorar la adecuación de los diferentes elementos de la web a las lenguas y culturas de los usuarios finales. Se han tomado como base dos do-

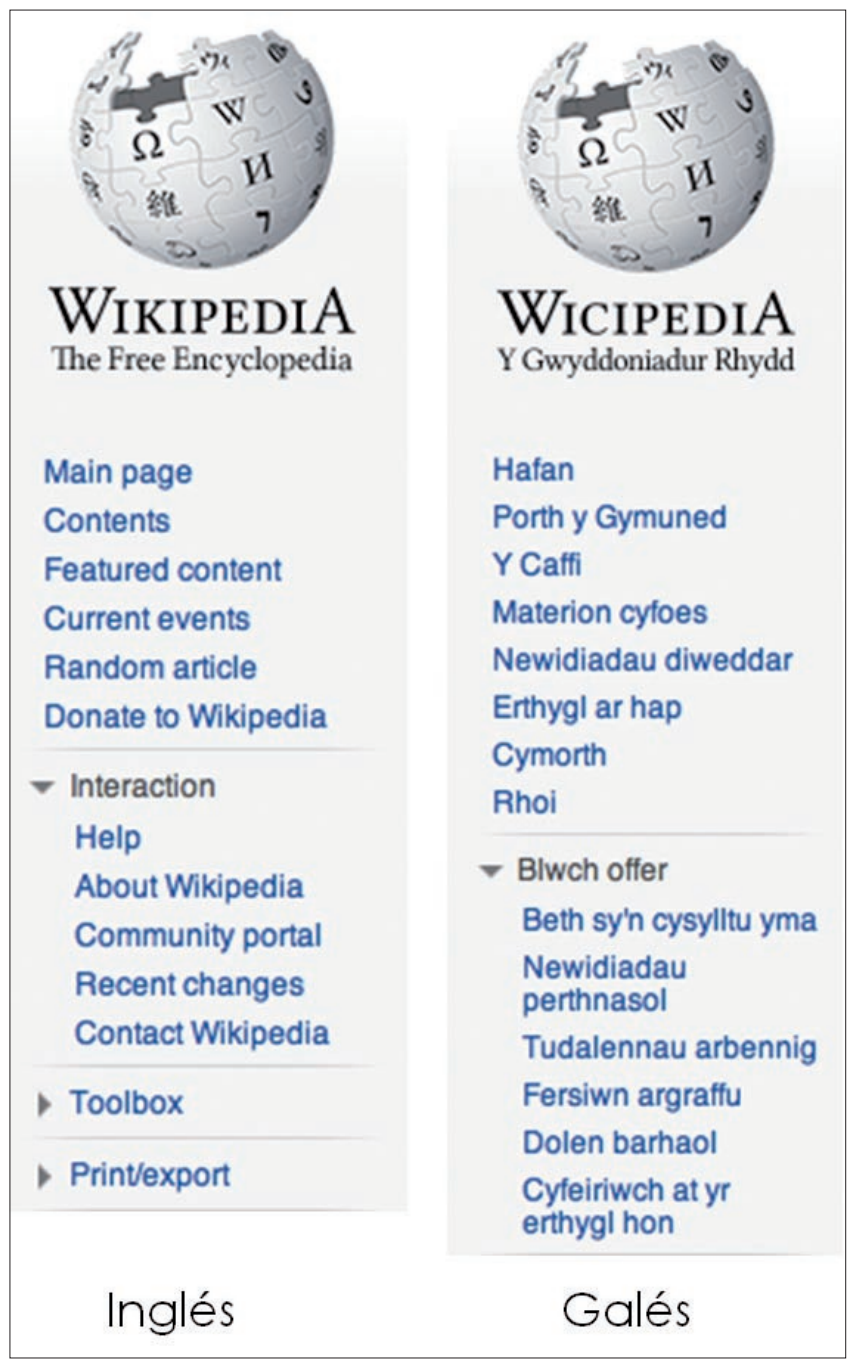

Figura 2. Ejemplo de dos versiones lingüísticas de Wikipedia con distinta longitud de palabras en el menú 


\begin{tabular}{|l|}
\hline Noticias \\
24/08/2011 Guatemala: Mujeres \\
indigenas padecen triple discriminación, \\
afirma ONU \\
24/08/2011 Economía palestina crece \\
gracias a ayuda extranjera: UNCTAD \\
24/08/2011 CEPAL pugna por impuesto a \\
transacciones financieras para apoyar \\
desarrollo
\end{tabular}

最新动态 RSS
'2011年8月24日 副秘书长米基罗赴
亚的斯亚贝巴出席非盟援助“非洲之
角”捐资会议

2011年8月24日 贸发会议：以色列 封锁严重抑制巴勒斯坦发展

\section{1年8月24日 环境署最新研究报 告：地球上有多少物种？870万种}

Figura 3. Ejemplo de cambio del formato de fecha en dos idiomas

cumentos del W3C para webs multilingües: Formatos de fechas y Fechas y horarios, y dos artículos (Marcus; Armitage; Frank, 1999) (Nielsen, 1996)

Pautas:

\subsection{Formato de fecha}

Debe considerarse cuál es la forma más común en el país al que se dirige la información, por ejemplo día-mes-año en España, pero año-mes-día en China. No adecuar el formato de fecha puede provocar confusión a la hora de interpretar la información. Un ejemplo sería la fecha de reserva de un vuelo, en la que el usuario quiera comprar un billete para el 1 de marzo y el sistema haga la reserva para el 3 de enero sin que el usuario se percate de que $01 / 03$ no es lo que él cree que es (figura 3).

Impacto: grave (3)

\subsection{Formato de hora}

Como ocurre con la fecha, la hora también cambia entre países. En unas culturas se utiliza la hora en formato de reloj de 12 horas mientras que en otras está más aceptado usar el reloj de 24 horas, de manera que en unos lugares es más común encontrar las 5PM y en otros las 17:00. Siguiendo con el ejemplo del vuelo, un usuario acostumbrado al sistema de 24 horas podía confundirse entre las 9 de la noche y las 9 de la mañana si no ve claramente que se refiere a las 21:00.

Impacto: grave (3)

\subsection{Formato de número}

De forma similar a los dos casos anteriores, la forma de leer la puntuación en las cifras cambia en función de la cultura. En unas lenguas la marca de millares es un punto (en español), en otros una coma (en inglés). Y pasa lo contrario con la marca de decimales, a veces es una coma (en español) y a veces un punto (en inglés). No adaptar la presentación de las cifras a la cultura del usuario puede provocar confusiones.

Impacto: grave (3)

\subsection{Sección de contacto}

A la hora de diseñar la página de contacto, hay que tener en cuenta si van a llegar a ella usuarios que viven en otros países. En tal caso, hay que proporcionar la información necesaria para contactar desde el extranjero, indicando datos como código de país para el teléfono, o si hay que incluir alguna cifra intermedia entre el código de país y el número local.

Impacto: grave (3)

2.5. Actualización del contenido en las diferentes versiones lingüísticas

Es muy común que un sitio web en varios idiomas no sea tan completo en todos ellos. Puede que uno de los idiomas sea el que se actualiza con más frecuencia, y en cambio otras versiones lingüísticas no cuenten con tanta dedicación para mantenerse al día. Lo ideal, sin duda, es que todas las versiones se actualicen al mismo tiempo.

Impacto: medio (2)

\subsection{Acceso desde cualquier página a la opción de elección de idioma}

Esta pauta se refiere a un aspecto de usabilidad, que es poder cambiar de idioma en cualquier página sin tener que comenzar a navegar desde la página de inicio al clicar la opción de cambio de lengua. Disponer de esta función bien implementada es tan sencillo como poner el link de la opción de idioma directamente a la página equivalente, y no dejar que siempre apunte a la home page. En cambio esto no es posible si el sitio no se ha traducido en tu totalidad; en tal caso, dirigir al usuario a la página de inicio es la solución más adecuada.

Impacto: grave (3)

Es importante traducir la etiqueta meta description porque los buscadores la usan a menudo para crear el snippet en la lista de resultados

\section{Grupo 3. Aspectos relacionados con los elementos visuales}

Teniendo en cuenta que los usuarios de diferentes culturas no reaccionan igual ante ciertos estímulos visuales (Makki; Leppert, 2006), es importante considerar la localización de los elementos visuales que se incluyan en el sitio, especial- 


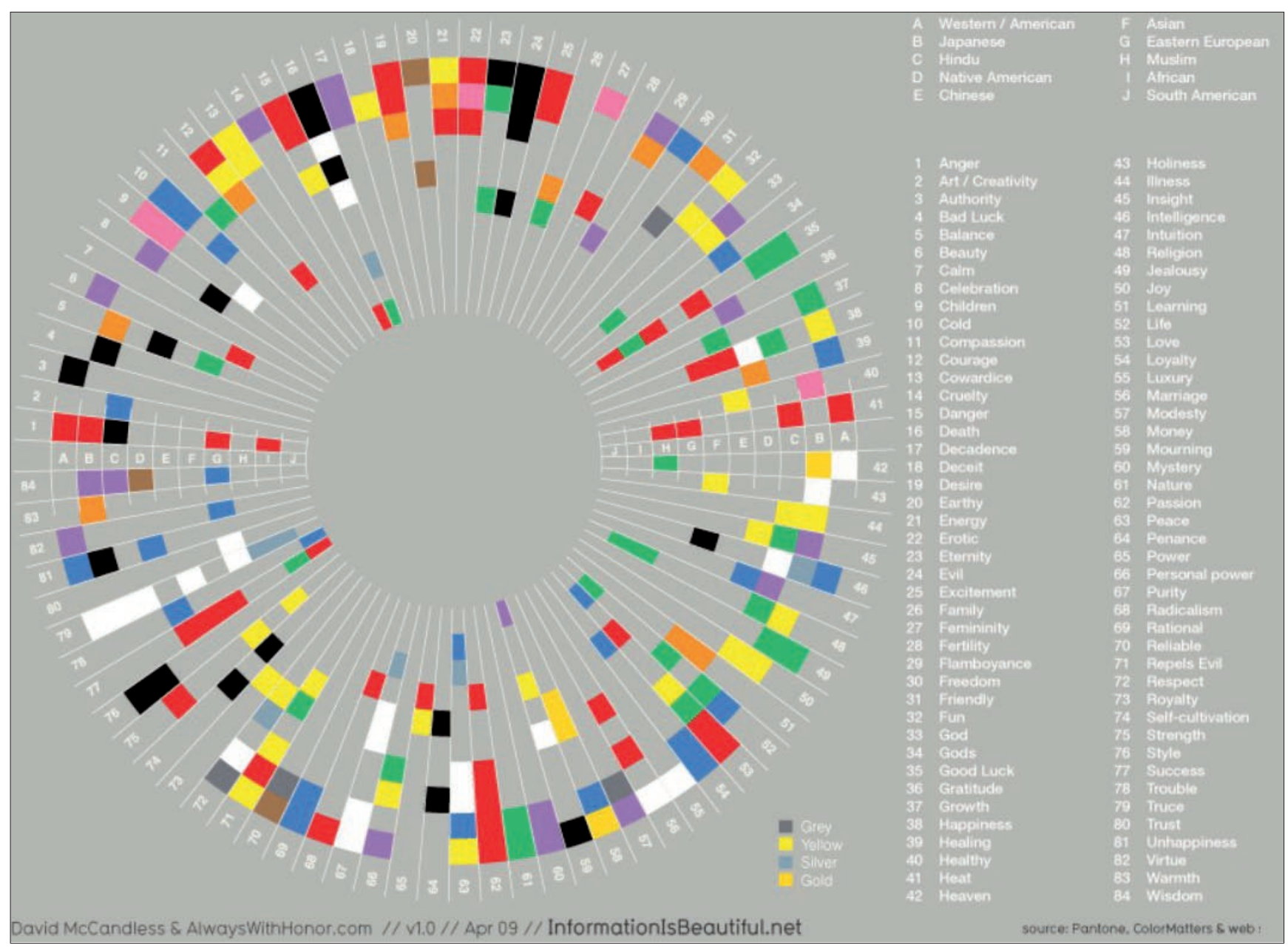

Figura 4. Distinto significado de los colores según las culturas, $h t t p: / / w w w . i n f o r m a t i o n i s b e a u t i f u l . n e t / v i s u a l i z a t i o n s /$ colours-in-cultures

mente imágenes, iconos y colores. Para los aspectos relacionados con la direccionalidad se ha tomado como fuente el documento del W3C sobre Dirección del sistema de escritura e idiomas.

\section{Pautas:}

\subsection{Iconos}

Los iconos deben adecuarse a cada cultura. Un ejemplo muy manido en usabilidad es el icono del buzón para recibir correo, tan distinto entre el español, normalmente cuadrado y plano, pegado a una pared o a una puerta, y el estadounidense, representado como un poste frente a la entrada de una casa con jardín, con un formato más cilíndrico.

Impacto: medio (2)

\subsection{Simbolismo de los colores}

Sobre el significado del color hay estudios muy interesantes que demuestran cómo colores que en unas culturas tienen un poso alegre en otros no lo tienen. La portada del libro Information is beautiful muestra un gráfico que ilustra muy bien esta idea:

http://www.informationisbeautiful.net/visualizations/colours-in-cultures

y nos hace pensar que el diseño visual usado en la versión dirigida a un país puede que tenga que variar con respecto al que se use para público de otros países.

Impacto: medio (2)

\subsection{Imágenes}

Aunque no suele ser un problema común, hay que tener en cuenta qué imágenes usar en un sitio web por si pueden tener connotaciones negativas u ofensivas para una cultura.

Impacto: medio (2)

\subsection{Direccionalidad}

La página web debe estar preparada para la lectura de izquierda a derecha y de derecha a izquierda, para los casos de traducciones a lenguas con diferente dirección, por ejemplo de inglés a árabe.

Impacto: grave (3)

\section{El instrumento de evaluación heurística está disponible en $h t t p: / / g 0 o . g l / L J T R 5$}

\section{Grupo 4. Aspectos que afectan a la búsqueda dentro del sitio web}

En caso de sitios web grandes, contar con un buscador interno resulta muy útil para localizar información sin tener que navegar. Si el buscador no ha sido implementado considerando que el sitio tiene información en diversos idiomas y que los usuarios pueden hacer las consultas también en varias lenguas, podrán darse problemas en la comprensión 
de los resultados obtenidos. En este punto se han seguido las indicaciones de Jakob Nielsen (1996).

\section{Pautas:}

\subsection{Búsqueda en todas las versiones lingüísticas}

El buscador debe recoger las páginas web de todo el sitio, no sólo las de un idioma en particular.

Impacto: medio (2)

\subsection{Opciones avanzadas de búsqueda relativas al idioma}

El usuario debe poder escoger si realiza una consulta en todos los idiomas o en alguno en particular.

Impacto: grave (3)

\subsection{Idioma de los textos informativos de la página de re- sultados}

Los textos que sirven de apoyo a los resultados de búsqueda deben aparecer en el idioma en el que el usuario esté consultando el sitio web. Esos textos son los que acompañan el listado y dan indicaciones de cómo mejorar la búsqueda, otras opciones para continuar navegando, etc.

Impacto: medio (2)

\section{Grupo 5. Aspectos que afectan al posicionamiento del sitio web en los buscadores}

Muchos sitios multilingües bien localizados no reciben el volumen de tráfico internacional que deberían porque no aparecen en los primeros resultados de las páginas de los buscadores. En esta categoría se evalúa si se han traducido los elementos relevantes para el posicionamiento web. Los indicadores desarrollados toman como fuente el artículo de Iler (2006). Es importante tener en cuenta que el posicionamiento de la página en cada uno de los idiomas es independiente, ya que los elementos relevantes de SEO (search engine optimization) son diferentes para cada versión lingüística.

\section{Pautas:}

\subsection{Traducción de la etiqueta title (head)}

Esta etiqueta corresponde al título de la página web, que es aquel que se lee en la parte superior del navegador y también el que identifica a la página web cuando aparece entre los resultados de búsqueda en los buscadores. Que esté traducido es importante para la identificación por parte de los usuarios, pero también para que los robots (spiders) de los buscadores puedan indexar ese título en el idioma en el que está escrita la página.

Impacto: grave (3)

\subsection{Traducción de las etiquetas description y keywords (head)}

Si bien estas etiquetas no son visibles desde el navegador, ya que se encuentran en la sección head del código fuente, los robots de los buscadores sí son capaces de leerlas e indexarlas. La etiqueta description tiene mucha importancia porque es la que en muchas ocasiones usan los buscadores para crear el resumen que dan en la lista de resultados (snippet). La etiqueta keywords en cambio no la utilizan los buscadores, por lo tanto en SEO no es relevante. Aun así, la hemos incluido en las pautas porque puede ser de utilidad disponer de ella para la implementación de un buscador interno del sitio web.

Impacto: grave (3)

\subsection{Traducción de las etiquetas alt y title de las imágenes}

Las imágenes no pueden ser indexadas por los buscadores porque no son legibles como texto. En cambio sí pueden indexar los textos vinculados a ellas, como ocurre con las etiquetas alt y title de las imágenes. Estas etiquetas, que indirectamente benefician al SEO por poder indexar información, son determinantes para que las personas ciegas puedan identificar el contenido de las imágenes con los programas especiales de lectura de páginas web.

Impacto: grave (3)

\subsection{Traducción de las etiquetas title de los enlaces}

De forma similar al punto anterior, la etiqueta title que acompaña a los enlaces permite dotar de información semántica complementaria a un elemento de la página web, que en este caso es el enlace a otra página. Esta etiqueta es visible a los ojos de los usuarios al pasar el cursor sobre el enlace, y también es visible a los robots de los buscadores.

Impacto: grave (2)

\section{Cómo utilizar esta checklist en la evaluación de la localización en sitios web multilingües}

A partir de las pautas de la sección anterior, se ha creado un instrumento de evaluación heurística (ver http://goo.gl/ LJTR5) en forma de tabla compuesto por:

- La lista de indicadores redactados en forma de pregunta.

- Una columna para indicar la frecuencia con la que se incurre en un error $(0=$ nunca, $1=$ a veces, $2=$ muchas veces o siempre).

- El impacto asociado a cada indicador (1 = leve, 2 = medio, $3=$ alto).

- Una columna donde calcular la severidad del error multiplicando el valor de frecuencia por el valor del impacto del indicador. La severidad indica la prioridad con la que un error debe ser subsanado. Su valor puede ir desde 0 hasta 6. La tabla 1 muestra las posibles combinatorias de frecuencia e impacto y el resultado de severidad para cada combinación.

- La fuente de donde se ha tomado el indicador.

Una vez que se cuenta con la heurística y sus indicadores, el procedimiento para llevar a cabo la evaluación sigue los pasos siguientes:

- Determinar el ámbito de aplicación de la evaluación, si se analizará todo el sitio web o alguna de las secciones.

- Disponer de un equipo de 3 a 5 evaluadores. Cada uno realiza el análisis de forma individual y redacta un informe con los resultados obtenidos.

- Poner en común los informes individuales: se jerarquizan los problemas en base a su severidad y se redacta un informe. 


\begin{tabular}{|c|c|l|}
\hline Frecuencia & Impacto & \multicolumn{1}{|c|}{ Severidad } \\
\hline \multirow{4}{*}{0} & 1 & $0=$ No hay error \\
\cline { 2 - 3 } & 2 & $0=$ No hay error \\
\cline { 2 - 3 } & 3 & $0=$ No hay error \\
\hline \multirow{4}{*}{1} & 1 & $1=$ Error muy leve \\
\cline { 2 - 3 } & 2 & $2=$ Error leve \\
\cline { 2 - 3 } & 3 & $3=$ Error de grado medio \\
\hline \multirow{3}{*}{2} & 1 & $2=$ Error leve \\
\cline { 2 - 3 } & 2 & $4=$ Prioritario \\
\cline { 2 - 3 } & 3 & $6=$ Urgente \\
\hline
\end{tabular}

Tabla 1. Grados de severidad calculados multiplicando los valores de frecuencia e impacto

Este método puede llevarse a cabo en cualquier momento del proceso de desarrollo de un sitio web o aplicación; sin embargo, se recomienda aplicarlo durante las primeras etapas de la elaboración de un producto, de manera que no se arrastren errores de usabilidad a lo largo del diseño. En caso de que se trate de un sitio web monolingüe que está preparando versiones en otros idiomas, lo ideal será aplicar una evaluación heurística específica para aspectos de localización antes de dar por acabada la traducción y adaptación del sitio. Una vez realizada la evaluación heurística y subsanados los problemas más graves que se han detectado, es aconsejable realizar un test con usuarios para completar la evaluación del sitio web.

\section{Nota}

1. A veces las palabras "internacionalización" y "localización" se abrevian con los numerónimos "i18n" (primera letra "i", última letra " $n$ " y en medio 18 letras más) y "I10n", respectivamente.

\section{Referencias}

Andreu-Vall, Mar; Marcos, Mari-Carmen. "Multilingüismo en la web. Pautas para un diseño centrado en el usuario". En: Interacción, 2011.

http://hdl.handle.net/10760/16407

Del-Galdo, Elisa; Nielsen, Jakob. International user interfaces. New York: John Wiley \& Sons, 1996.

Griho (Grupo de Investigación en Interacción Persona Ordenador e Integración de Datos). "Métodos de evaluación de la usabilidad". En: Modelo de proceso de la ingeniería de la usabilidad y de la accesibilidad. MPlu+a, 2010.

http://www.grihohcitools.udl.cat/mpiua/evaluacionusabili dad.htm

Hassan-Montero, Yusef; Martín-Fernández, Francisco J. "Guía de evaluación heurística de sitios web", No sólo usabilidad, 2003.

http://www.nosolousabilidad.com/articulos/heuristica.htm

Hillier, Mathew. "The role of cultural context in multilingual website usability". Journal of electronic commerce research and applications, 2002, v. 2, n. 1, pp. 2-14.
http://dx.doi.org/10.1016/S1567-4223(03)00005-X

Iler, Huiping. Maximizing visibility for multilingual web sites, 2006, wintranslation.com

http://www.sempo.org/resource/resmgr/Docs/wintransla tion_for_SEMPO.pdf

Internet WorldStats. International world users by language. Top 10 languages, 2010.

http://www.internetworldstats.com/stats7.htm

Makki, Kami; Leppert, Greg. "Factors of usability design for multilingual and multicultural websites". En: IRI, 2006, pp. 6-10.

http://dx.doi.org/10.1109/IRI.2006.252379

Marcus, Aaron; Armitage, John; Frank, Volker. Globalization of user-interface design for the web. Report of human factors \& the web, 1999.

http://www.amanda.com/cms/uploads/media/AMA_Glo balizationUserInterfaceDesignWeb.pdf

Nielsen, Jakob. How to conduct a heuristic evaluation, 1994. http://www.useit.com/papers/heuristic/heuristic_evalua tion.html

Nielsen, Jakob. International web usability. Alertbox, 1996. http://www.useit.com/alertbox/9608.html

Nielsen, Jakob. "Utilización internacional: atender a una audiencia global”. En: Nielsen, Jakob. Usabilidad: diseño de sitios web. Madrid: Pearson Education, 2000.

Nielsen, Jakob; Mack, Robert. (eds.). Usability inspection methods. New York: John Wiley \& Sons, 1994.

Nielsen, Jakob; Molich, Rolf. "Heuristic evaluation of user interfaces". En: CHI, 1990, pp. 249-256.

W3C. Dirección del sistema de escritura e idiomas. http://www.w3.org/International/questions/qa-scripts

W3C. El tamaño del texto en la traducción. http://www.w3.org/International/articles/article-text-size

W3C. Etiquetas de idioma en html y $\mathrm{xml}$. http://www.w3.org/International/articles/language-tags

W3C. Fechas y horarios.

http://www.w3.org/International/O-time

W3C. Formatos de fechas.

http://www.w3.org/International/questions/qa-date-for mat

W3C. Guía breve de internacionalización.

http://www.w3c.es/divulgacion/guiasbreves/internaciona lizacion

W3C. Identifying the language of a target document. http://www.w3.org/International/geo/html-tech/techlang.html\#ri20050128.152033553

W3C. Internationalization (I18n) activity. http://www.w3.org/International

W3C. Introducción a direcciones web plurilingües. http://www.w3.org/International/articles/idn-and-iri 